\title{
A Reformulation of Newton Second Law for Charged Particles and Its Application to Quantum Dynamics
}

\author{
Vu B. Ho \\ 9 Adela Court, Mulgrave, Australia \\ Email: vubho@bigpond.net.au
}

How to cite this paper: Ho, V.B. (2021) A Reformulation of Newton Second Law for Charged Particles and Its Application to Quantum Dynamics. Journal of Applied Mathematics and Physics, 9, 2796-2806. https://doi.org/10.4236/jamp.2021.911180

Received: October 18, 2021

Accepted: November 14, 2021

Published: November 17, 2021

Copyright $\odot 2021$ by author(s) and Scientific Research Publishing Inc. This work is licensed under the Creative Commons Attribution International License (CC BY 4.0).

http://creativecommons.org/licenses/by/4.0/

\section{(c) (i) Open Access}

\begin{abstract}
We propose a reformulation of Newton's second law of motion for charged particles and possible applications of the reformulation to quantum dynamics. We show that the negative energy states arising from the Dirac equation in relativistic quantum mechanics can be verified using the reformulating framework. We also discuss possible hidden dynamics underlying the concept of quantum jumps in quantum mechanics as outlined in Schrödinger's article: ARE THERE QUANTUM JUMPS? In this case, we show that the hidden dynamics of quantum jumps are also determined by the Coulomb interaction between charged particles.
\end{abstract}

\section{Keywords}

Reformulation of Newton Second Law, Dirac Negative Energy States, Quantum Jumps in Quantum Mechanics

\section{Introduction}

The development of physical theories has been based mainly on the results of experimental measurements of physical processes, and the experimental results themselves depend not only on the quality of the instruments that are available at the time but also on the concepts and perceptions of physical objects. Consequently, physical theories have been changed and reformulated to explain new physical phenomena that cannot be explained by the previous theories. However, even with the highly developed modern theories of quantum mechanics, with attempts to explain physical phenomena that seem to contradict with classical theories, basically the foundation of quantum physical theories still remains, which is Newton's second law of motion in which the concept of inertial mass 
plays the fundamental role in the determination of the dynamics of a particle under the influence of a force caused by a physical field [1] [2]. At the time when Newton formulated the classical dynamics, scientists were mainly studying the dynamics of macroscopic objects related to their weight, therefore it was reasonable for those scientists to suggest the physical object that plays the fundamental role in dynamical equations of motion is the inertial mass. However, we may ask why the inertial mass alone should play such a fundamental role in determining the dynamics of physical objects under the influence of forces caused by other physical fields, such as the electromagnetic field. At the microscopic quantum scale, it is obvious that the mass and the charge of an elementary particle are closely related, hence they should be treated equally fundamentally in physical theories to deal with quantum physical phenomena. For example, in relativistic quantum mechanics, Dirac equation predicts the existence of a positron on the same footing as that of an electron but the subsequent description of the existence for the positron is completely different from that of the electron, such as in terms of Dirac Sea or moving backward in time, even though the instruments used to detect the positron are almost identical to that for the electron. A distinct problem associated with Dirac equation is the existence of negative energy states that should not exist according to the definition of kinetic energy within the current formulation of classical dynamics based on Newton's second law of motion. This is in fact one of the main topics discussed in this work that led to the need to reformulate Newton's second law so that a new definition of kinetic energy would allow assigning both positive and negative values to it. Another interesting topic that we will discuss using the reformulated Newton's second law of motion is related to the concept of quantum jumps in quantum mechanics. In quantum mechanics, a quantum jump is a discontinuous transition from one quantum state to another. Such a process of transition is profoundly different from the continuous transition between states in physical systems described in classical physics. Schrödinger, one of the founders of quantum mechanics, asked the question: ARE THERE QUANTUM JUMPS? And he expressed his view: "There have been ingenious constructs of the human mind that gave an exceedingly accurate description of observed facts and have yet lost all interest except to historians. I am thinking of the theory of epicycles. I confess to the heretical view that their modern counterpart in physical theory are the quantum jumps" [3]. It is clear from such view of the founder of wave mechanics that we would need a radical change in the classical formulation of physics that would allow a description of quantum jumps in terms of classical dynamics. And a hidden dynamics underlying the quantum jumps should also be determined by interactions between physical objects associated with elementary particles.

\section{Reformulation of Newton's Second Law of Motion}

We now reformulate Newton's second law of motion, $\boldsymbol{F}=m \boldsymbol{a}$, in which the 
inertial mass $m$ of a charged particle will be replaced by the ratio of inertial mass $m$ to charge $q$ of the particle, $M=m / q$. Let us image that the modern scientific investigation of natural phenomena that involve elementary particles started with J. J. Thomson experiment, which discovered the electron by determining its ratio of mass $m$ to charge $q$ [4]. It was found from the Thomson experiment that the quantity $M=m / q$, electric field $\boldsymbol{E}$, and acceleration $\boldsymbol{a}$ of the electron satisfy the equation

$$
M a=E
$$

If we consider Equation (1) as a fundamental dynamical equation of motion of the electron under the electric field $\boldsymbol{E}$ then the quantity $M$ should also be considered as a fundamental physical object that would enter all dynamical equations resulted from further investigations of the dynamics of the electron when it interacts with other elementary particles through physical fields.

For a particle with an inertial mass $m$ and a charge $q$ moving with velocity $\boldsymbol{v}=\mathrm{d} \boldsymbol{r} / \mathrm{d} t$, we define a reformulated linear momentum $\boldsymbol{p}$ in terms of the physical object $M=m / q$ and the velocity $\boldsymbol{v}$ as

$$
p=M v
$$

If the inertial mass $m$ is always positive then the sign of the quantity $M$ depends solely on the sign of the charge $q$. Also, as in Newtonian mechanics, we introduce the concept of force $\boldsymbol{F}$ exerted on the particle according to the differential equation

$$
\boldsymbol{F}=\frac{\mathrm{d} \boldsymbol{p}}{\mathrm{d} t}=M \frac{\mathrm{d} \boldsymbol{v}}{\mathrm{d} t}=M \frac{\mathrm{d}^{2} \boldsymbol{r}}{\mathrm{d} t^{2}}=M \boldsymbol{a}
$$

Then, in terms of physical objects $M_{1}=m_{1} / q_{1}$ and $M_{2}=m_{2} / q_{2}$, and the force law given in Equation (3), Newton's universal law of gravitation can be rewritten in the form

$$
M_{1} \boldsymbol{a}=g \frac{M_{1} M_{2}}{r^{3}} \boldsymbol{r}
$$

where the value of the dimensional constant $g$ is $g=G q_{2}$. And Coulomb's law can also be rewritten in terms of the physical objects $M_{1}$ and $M_{2}$ as

$$
M_{1} \boldsymbol{a}=K \frac{q_{2}}{r^{3}} \boldsymbol{r}=k \frac{1}{M_{2}} \frac{1}{r^{3}} \boldsymbol{r}
$$

where $k=K m_{2}$.

In the reformulating framework, we also introduce the fundamental concept of work done $W$ by a force $\boldsymbol{F}$ according to the integral

$$
W=\int \boldsymbol{F} \mathrm{d} \boldsymbol{s}=\int M \frac{\mathrm{d} \boldsymbol{v}}{\mathrm{d} t} \cdot \boldsymbol{v} \mathrm{d} t=\frac{M}{2} \int \frac{\mathrm{d}}{\mathrm{d} t}\left(v^{2}\right) \mathrm{d} t
$$

The scalar quantity $T=M v^{2} / 2$ obtained from Equation (6) will also be identified as the kinetic energy. With this definition, depending on the sign of the charge $q$, the kinetic energy can take either positive or negative value. For example, if the inertial mass of both an electron and a positron is positive then, using 
the current convention, the electron has a negative kinetic energy, but the kinetic energy of the positron is positive. For a conservative force defined in terms of a potential $V$ by the relation $\boldsymbol{F}=-\nabla V$ we then again have

$$
W=\int \boldsymbol{F} \mathrm{d} \boldsymbol{s}=-\int \nabla V \mathrm{~d} \boldsymbol{s}
$$

From Equations (6) and (7), the total energy $E$ can be obtained

$$
E=T+V=\frac{1}{2} M v^{2}+V
$$

So far, except for the new dimensions associated with physical entities involved, basically the reformulated mathematical formulation of classical mechanics remains the same as that of Newtonian physics. However, as shown below, the reformulated Newton's second law of motion in fact has profound consequences that involve the quantum dynamics of elementary particles.

\section{Reformulation of Hamiltonian}

Following the Hamiltonian formulation in classical physics, we define a reformulated Hamiltonian for a conservative system as the total energy expressed in terms of the physical object $M=m / q$, reformulated linear momentum $\boldsymbol{p}$ and potential $V$ in the form

$$
H=\frac{\boldsymbol{p}^{2}}{2 M}+V
$$

From the dimension of the kinetic term given in Equation (9), the physical unit of the reformulated Hamiltonian is J/C (Joule per Coulomb). It should be emphasised here that, as shown in Equation (5) for Coulomb's law of two charged particles, the potential $V$ depends only on the charge of the particle considered to be the source of interaction. For a system of two particles with physical objects $M_{1}$ and $M_{2}$, which interact through a potential $V\left(\boldsymbol{r}_{2}-\boldsymbol{r}_{1}\right)$ depending only on the relative positions of the two particles, the Hamiltonian of the system can be written as

$$
H\left(\boldsymbol{r}_{1}, \boldsymbol{r}_{2}\right)=\frac{p_{1}^{2}}{2 M_{1}}+\frac{p_{2}^{2}}{2 M_{2}}+V\left(\boldsymbol{r}_{2}-\boldsymbol{r}_{1}\right)
$$

It is observed that since the physical objects $M_{1}$ and $M_{2}$ can take positive or negative values therefore if the motion of the two charged particles in the system so that the dynamical condition $p_{2}^{2} / M_{2}=-p_{1}^{2} / M_{1}$ is satisfied then the reformulated Hamiltonian given in Equation (10) reduces to

$$
H\left(\boldsymbol{r}_{1}, \boldsymbol{r}_{2}\right)=V\left(\boldsymbol{r}_{2}-\boldsymbol{r}_{1}\right)
$$

In this case the dynamics of such system is determined only by the interacting potential between the two charged particles. In particular, for the system of a particle and its corresponding antiparticle, such as an electron and a positron, we have $M_{2}=-M_{1}$. If the dynamics of these two particles is symmetric then we can assume further that $p_{1}^{2}=p_{2}^{2}$, and the Hamiltonian given in Equation (10) also reduces to Equation (11). Hence, the kinetic energies of such two particles 
cancel each other out and the corresponding Hamiltonian describing the dynamics of the system depends only on the potential and, consequently, the inertial mass is no longer associated with the dynamics of such system. The physical objects do not manifest dynamically as particles but only in the form of interacting potential depending on the charge.

Also, following the usual method of canonical quantisation in quantum mechanics [5], if we apply a modified quantisation procedure

$$
p=M v=\frac{m v}{q} \rightarrow-i \frac{\hbar}{q} \frac{\partial}{\partial x}, \quad E \rightarrow i \frac{\hbar}{q} \frac{\partial}{\partial t}
$$

then the Hamiltonian given in Equation (9) takes the quantisation form

$$
H=-\frac{\hbar^{2}}{2 M q^{2}} \nabla^{2}+V
$$

and the quantum formulation of the Hamiltonian given in Equation (10) becomes

$$
H\left(\boldsymbol{r}_{1}, \boldsymbol{r}_{2}\right)=-\frac{\hbar^{2}}{2 M_{1} q_{1}^{2}} \nabla_{r_{1}}^{2}-\frac{\hbar^{2}}{2 M_{2} q_{2}^{2}} \nabla_{r_{2}}^{2}+V\left(\boldsymbol{r}_{2}-\boldsymbol{r}_{1}\right)
$$

Equation (14) can also be rewritten in terms of the relative position $\boldsymbol{r}=\boldsymbol{r}_{2}-\boldsymbol{r}_{1}$ and the quantity $\boldsymbol{R}$ defined as

$$
\boldsymbol{R}=\frac{M_{1} \boldsymbol{r}_{1}+M_{2} \boldsymbol{r}_{2}}{M_{1}+M_{2}}
$$

The quantity $\boldsymbol{R}$ may be identified as the position of the centre of mass of the system. In terms of the relative position $\boldsymbol{r}$ and quantity $\boldsymbol{R}$, Equation (14) converts into the form

$$
\begin{aligned}
H(\boldsymbol{r}, \boldsymbol{R})= & -\frac{\hbar^{2}}{2\left(M_{1}+M_{2}\right)^{2}}\left(\frac{M_{1}}{q_{1}^{2}}+\frac{M_{2}}{q_{2}^{2}}\right) \nabla_{\boldsymbol{R}}^{2}+\frac{\hbar^{2}}{M_{1}+M_{2}}\left(\frac{1}{q_{2}^{2}}-\frac{1}{q_{1}^{2}}\right) \nabla_{\boldsymbol{R} r}^{2} \\
& -\frac{\hbar^{2}}{2}\left(\frac{1}{M_{1} q_{1}^{2}}+\frac{1}{M_{2} q_{2}^{2}}\right) \nabla_{\boldsymbol{r}}^{2}+V(\boldsymbol{r})
\end{aligned}
$$

It should be mentioned here that the description using the centre of mass system according to the transformation given in Equation (15) cannot be applied to a system consisting of a particle and its corresponding antiparticle, since in this case we have $M_{2}=-M_{1}$.

\section{On Negative Energy States Associated with Dirac Equation}

Dirac formulated his relativistic wave equation to describe the dynamics of a free electron by using the relativistic relation $E^{2}=c^{2} p^{2}+m^{2} c^{4}$ between the total energy $E$, momentum $p$, rest mass $m$, and speed of light in vacuum $c$. A fundamental problem related to using this relativistic relation is that according to the formulation of classical physics values of the energy $E$ associated with a free particle are positive, however the wavefunctions obtained from Dirac equation can be associated with negative energy states [6]. We now show that this problem 
can be avoided within the reformulating framework. The relativistic momentum $\boldsymbol{p}$ and energy $E$ of a moving object in the reformulating framework can be found as

$$
\begin{aligned}
& \boldsymbol{p}=\frac{M_{0} \boldsymbol{v}}{\sqrt{1-v^{2} / c^{2}}} \\
& E=\frac{M_{0} c^{2}}{\sqrt{1-v^{2} / c^{2}}}
\end{aligned}
$$

where $M_{0}=m_{0} / q$, with $m_{0}$ and $q$ are the rest mass and the charge of a particle, respectively. From Equations (17) and (18), we obtain the following relation

$$
E^{2}=c^{2} p^{2}+M_{0}^{2} c^{4}
$$

Using the relativistic energy-momentum relation given in Equation (19), a reformulated Dirac equation can be derived as

$$
i(\hbar / q) \gamma^{\mu} \partial_{\mu} \psi-M_{0} c \psi=0
$$

where the gamma matrices $\gamma^{\mu}$ and the Pauli matrices $\sigma^{\mu}$ are given as follows

$$
\begin{gathered}
\gamma^{k}=\left(\begin{array}{cc}
0 & \sigma_{k} \\
-\sigma_{k} & 0
\end{array}\right), \quad \gamma^{0}=\left(\begin{array}{cc}
1 & 0 \\
0 & -1
\end{array}\right) \\
\sigma_{1}=\left(\begin{array}{ll}
0 & 1 \\
1 & 0
\end{array}\right), \quad \sigma_{2}=\left(\begin{array}{cc}
0 & -i \\
i & 0
\end{array}\right), \quad \sigma_{3}=\left(\begin{array}{cc}
1 & 0 \\
0 & -1
\end{array}\right)
\end{gathered}
$$

In particular, the reformulated Dirac equation for a stationary particle, $\boldsymbol{p}=0$, takes the form

$$
i \frac{\hbar}{q} \frac{\partial \Psi}{\partial t}=M_{0} c^{2} \gamma^{0} \Psi
$$

It is observed that using the definition $M_{0}=m_{0} / q$, Equation (23) can be reduced to the original Dirac equation $i \hbar \partial \Psi / \partial t=m_{0} c^{2} \gamma^{0} \Psi$ for a stationary particle. However, using this original Dirac equation will lead to the problem of negative energy states associated with the negative rest energy $E=-m_{0} c^{2}$. On the other hand, from Equation (23) with the reformulated rest energy $E=M_{0} c^{2}$ we obtain two solutions, which can be written in the form

$$
\Psi_{1}=\left(\begin{array}{l}
1 \\
0 \\
0 \\
0
\end{array}\right) \mathrm{e}^{-i M_{0} c^{2} t /(\hbar / q)}, \quad \Psi_{2}=\left(\begin{array}{l}
0 \\
1 \\
0 \\
0
\end{array}\right) \mathrm{e}^{-i M_{0} c^{2} t /(\hbar / q)}
$$

Again, using Equation (23) with $E=-M_{0} c^{2}$ we also obtain two solutions, which can be written in the form

$$
\Psi_{3}=\left(\begin{array}{l}
0 \\
0 \\
1 \\
0
\end{array}\right) \mathrm{e}^{i M_{0} c^{2} t /(\hbar / q)}, \quad \Psi_{4}=\left(\begin{array}{l}
0 \\
0 \\
0 \\
1
\end{array}\right) \mathrm{e}^{i M_{0} c^{2} t /(\hbar / q)}
$$

Since in the reformulation of Newton's second law of motion the reformu- 
lated rest energy $E=M_{0} c^{2}$ can take positive or negative values depending on the sign of the charge of a particle, therefore there should be no question regarding the negative energy states.

\section{Hidden Dynamics of Quantum Jumps in a Hydrogen Atom}

In this section we analyse two possible dynamics associated with a hydrogen atom, one of which may be identified with hidden dynamics of quantum jumps. The first dynamics is the usual dynamics of the physical system consisting of an electron and a proton. The second dynamics of the system whose Hamiltonian, on the other hand, depends only on the Coulomb potential shown in Equation (11).

\subsection{Schrödinger Dynamics of a Hydrogen Atom}

For a hydrogen atom consisting of a proton with physical object $M_{1}=m_{p} / q$ and an electron with physical object $M_{2}=m_{e} /(-q)$, the Hamiltonian given in Equation (16) reduces to

$$
H(\boldsymbol{r}, \boldsymbol{R})=-\frac{\hbar^{2}}{2 q\left(m_{p}-m_{e}\right)} \nabla_{\boldsymbol{R}}^{2}-\frac{\hbar^{2}}{2 q}\left(\frac{1}{m_{p}}-\frac{1}{m_{e}}\right) \nabla_{r}^{2}+V(\boldsymbol{r})
$$

where the reduced Coulomb potential $V=K q / r$ depending only on the charge of the proton, as shown in Equation (5). Working in the centre of mass system, the Schrödinger equation for the dynamics of the electron with charge $-q$ takes the form

$$
i \frac{\hbar}{-q} \frac{\partial \Psi}{\partial t}=-\frac{\hbar^{2}}{2 q}\left(\frac{1}{m_{p}}-\frac{1}{m_{e}}\right) \nabla^{2} \Psi+K \frac{q}{r} \Psi
$$

Equation (27) can be rewritten as in the original form of the time-dependent Schrödinger equation for a hydrogen atom [7]

$$
i \hbar \frac{\partial \Psi}{\partial t}=-\frac{\hbar^{2}}{2 \mu_{r}} \nabla^{2} \Psi-K \frac{q^{2}}{r} \Psi
$$

However, in Equation (28) the reduced mass is defined as

$\mu_{r}=m_{p} m_{e} /\left(m_{p}-m_{e}\right)$. In general, the reformulated reduced mass $\mu_{r}$ is very different from the usual reduced mass defined by $\mu_{r}=m_{p} m_{e} /\left(m_{p}+m_{e}\right)$. Though, as shown below for the case of the hydrogen atom, since $m_{p} \gg m_{e}$, the values of the energy levels obtained from Schrödinger equation are almost identical when evaluated using either of these two types of reduced mass. The Schrödinger equation given in Equation (28) admits stationary state solutions

$$
\Psi=\psi \exp (-i E t / \hbar)
$$

where $E$ is the expectation value of the total energy. The wavefunction $\psi$ then satisfies the time-independent Schrödinger equation

$$
-\frac{\hbar^{2}}{2 \mu_{r}} \nabla^{2} \psi-K \frac{q^{2}}{r} \psi=E \psi
$$


Solutions to Equation (30) in spherical polar coordinates can be found in terms of spherical harmonics and radial functions $\psi_{E l m}(r, \theta, \phi)=R_{n l}(r) Y_{l m}(\theta, \phi)$. The functions $R_{n l}(r)$ and $Y_{l m}(\theta, \phi)$ are given as

$$
\begin{aligned}
& Y_{l m}(\theta, \phi)=(-1)^{m}\left(\frac{(2 l+1)(l-m) !}{4 \pi(l+m) !}\right)^{1 / 2} P_{l}^{m}(\cos \theta) \mathrm{e}^{i m \phi} \\
& R_{n l}(r)=-\left(\left(\frac{2}{n a_{0}}\right)^{3} \frac{(n-l-1) !}{2 n((n+l) !)^{3}}\right)^{1 / 2} \mathrm{e}^{-\rho / 2} \rho^{l} L_{n+l}^{2 l+1}(\rho)
\end{aligned}
$$

where $\rho=\left(2 / n a_{0}\right) r$, and $a_{0}=4 \pi \varepsilon_{0} \hbar^{2} / \mu_{r} q^{2}$. The energy levels $E_{n}$ associated with the stationary states of the electron are found as

$$
E_{n}=-\frac{\mu_{r}}{2 \hbar^{2}}\left(\frac{q^{2}}{4 \pi \varepsilon_{0}}\right)^{2} \frac{1}{n^{2}}
$$

Using the recommended values of the fundamental physical constants [8], the values of the energy levels with the reformulated reduced mass $\mu_{r}=m_{p} m_{e} /\left(m_{p}-m_{e}\right)$ can be found as $E_{n}=-13.61 / n^{2} \mathrm{eV}$. On the other hand, for the reduced mass $\mu_{r}=m_{p} m_{e} /\left(m_{p}+m_{e}\right)$, the energy levels are $E_{n}=-13.60 / n^{2} \mathrm{eV}$.

\subsection{Potential Hidden Dynamics in a Hydrogen Atom}

To Schrödinger, even though their physical nature still remains a mystery, the wavefunctions representing the states of the electron of a hydrogen atom should behave according to the laws of classical physics. There should be a hidden dynamics that may be used to describe quantum jumps as continuous transitions between states of a quantum dynamical system. With this perception about the nature of the wavefunctions, Schrödinger attempted to explain classically the dynamics of quantum jumps after inventing his own theory of wave mechanics. Once again, we quote the explanation using his own words as follows [3]:

"The achievement of wave mechanics was, that it found a general model picture in which the 'stationary' states of Bohr's theory take the role of proper vibrations, and their discrete 'energy levels' the role of the proper frequencies of these proper vibrations; and all this follows from the new theory, once it is accepted, as simply and neatly as in the theory of elastic bodies, which we mentioned as a simile. Moreover, the radiated frequencies, observed in the line spectra, are in the new model, equal to the differences of the proper frequencies; and this is easily understood, when two of them are acting simultaneously, on simple assumptions about the nature of the vibrating 'something'.

But to me the following point has always seemed the most relevant, and it is the one I wish to stress here, because it has been almost obliterated - if words mean something, and if certain words now in general use are taken to mean what they say. The principle of superposition not only bridges the gaps between the 'stationary' states, and allows, nay compels us, to admit intermediate states without removing the discreteness of the 'energy levels' (because they have be- 
come proper frequencies); but it completely does away with the prerogative of the stationary states. The epithet stationary has become obsolete. Nobody who would get acquainted with wave mechanics without knowing its predecessor (the Planck-Einstein-Bohr-theory) would be inclined to think that a wave-mechanical system has a predilection for being affected by only one of its proper modes at a time. Yet this is implied by the continued use of the words 'energy levels', 'transitions', 'transition probabilities'.

The perseverance in this way of thinking is understandable, because the great and genuine successes of the idea of energy parcels have made it an ingrained habit to regard the product of Planck's constant $h$ and a frequency as a bundle of energy, lost by one system and gained by another. How else should one understand the exact dove-tailing in the great 'double-entry' book-keeping in nature? I maintain that it can in all cases be understood as a resonance phenomenon."

From Schrödinger's arguments about the nature of the wavefunctions, it is reasonable to suggest, intuitively, that when specifying the proper modes of vibration for physical system of an atom, Schrödinger equation establishes a mechanism to determine a spatial structure of the atom with definite energy levels to deal with the electromagnetic field. The stationary states are part of a mechanism that controls the process of radiation of photons, which is a resonance set up between two stationary states. Therefore, from the view of classical dynamics, there must be internal dynamics that allow such resonance to be established in the process of radiation and such dynamics may be identified as hidden dynamics of quantum jumps. For the situation when a hydrogen atom absorbs the energy of an electromagnetic field from outside, besides the dynamics described by the Schrödinger equation as discussed above, we suggest that the absorbed energy may cause internal dynamics to the system of the electron and the proton, which could be in the form of an oscillation so that these internal dynamics satisfy the dynamical condition

$$
\frac{p_{2}^{2}}{M_{2}}=-\frac{p_{1}^{2}}{M_{1}} \text { or } \frac{v_{e}}{v_{p}}=\sqrt{\frac{m_{p}}{m_{e}}}
$$

In this case, the Hamiltonian given in Equation (10) reduces to the Hamiltonian given in Equation (11), and the Schrödinger equation for the internal dynamics of the electron now takes the form

$$
i \frac{\hbar}{-q} \frac{\partial \Psi}{\partial t}=K \frac{q}{r} \Psi
$$

Let $v=K q^{2} /(\hbar r)$ then we obtain the solution

$$
\Psi=\Psi_{0}(\boldsymbol{r}) \mathrm{e}^{i v t}
$$

where $\Psi_{0}(\boldsymbol{r})$ is an arbitrary function, and the potential determines the frequency of a standing wave, resulted from "something" vibrating as stated by Schrödinger in the above quotation, which provides a medium for resonances to occur between stationary states to produce photons. In general, any system consisting of two charged particles of opposite signs may set up a standing wave 
whose frequency at a position in space is determined by the value of the Coulomb potential at that position. Therefore, without specifying what is really vibrating, we may suggest that these particles can turn space into a dynamical system and space itself oscillates from the result of superposition of standing waves. Space therefore can be endowed with a geometric, and topological, structure which manifests in the form of a potential of a physical field [9] [10] [11] [12]. For the case of a physical system whose dynamics is determined by Schrödinger equation, solutions given in Equation (36) can be identified as the hidden dynamics of quantum jumps between stationary states. Overall, atoms are provided with this mechanism to absorb the electromagnetic energy to create photons, or to absorb photons and then release them. The electromagnetic energy may be continuous, but energies carried by photons are discrete. Epistemologically, stationary states with their corresponding energy levels may be thought of as being designed so that particular amounts of energy can be created.

\section{Conclusion}

In this work, we reformulated Newton's second law of motion for charged particles in which not only the inertial mass, as in the original formulation of Newton's second law but also the charge of a particle plays the fundamental role in inertial reaction to motion. We discussed possible applications of the reformulation to quantum dynamics, such as verifying the negative energy states arising from Dirac equation in relativistic quantum mechanics by using a reformulated relativistic energy-momentum relation, or showing possible underlying dynamics related to the concept of quantum jumps in quantum mechanics as outlined in Schrödinger's article: ARE THERE QUANTUM JUMPS? For the case of quantum jumps, we showed that hidden dynamics of quantum jumps could simply be a result of Coulomb interaction in which charged particles do not manifest dynamically as particles but only in the form of interacting potential.

\section{Acknowledgements}

We would like to thank the reviewers for their constructive comments, and we would also like to thank the administration of JAMP for their editorial advice during the preparation of this work.

\section{Conflicts of Interest}

The author declares no conflicts of interest regarding the publication of this paper.

\section{References}

[1] Newton, I. (1846) The Mathematical Principles of Natural Philosophy. Translated into English by Andrew Motte, Published by Daniel Adee, New York.

[2] Goldstein, H., Poole, C. and Safko, J. (2000). Classical Mechanics. Addison Wesley, New York. 
[3] Schrödinger, E. (1952) Are There Quantum Jumps? Part I. The British Journal for the Philosophy of Science, 3, 109-123. https://doi.org/10.1093/bjps/III.10.109

[4] Anderson, E.E. (1982) Introduction to Modern Physics. Saunders College Publishing, New York.

[5] Bransden, B.H. and Joachain, C.J. (1989) Introduction to Quantum Mechanics. Longman Scientific \& Technical, New York.

[6] Dirac, P.A.M. (1928) The Quantum Theory of the Electron. Proceedings of the Royal Society A: Mathematical, Physical and Engineering Sciences, 117, 610-624. https://doi.org/10.1098/rspa.1928.0023

[7] Schrödinger, E. (1982) Collected Papers on Wave Mechanics. AMS Chelsea Publishing, New York.

[8] Mohr, P.J. and Taylor, B.N. (2000) CODATA Recommended Values of the Fundamental Physical Constants. Reviews of Modern Physics, 72, Article No. 351. https://doi.org/10.1103/RevModPhys.72.351

[9] Ho, V.B. (2018) Spacetime Structures of Quantum Particles. International Journal of Physics, 6, 105-115. https://doi.org/10.12691/ijp-6-4-2

[10] Ho, V.B. (2018) A Classification of Quantum Particles. Global Journal of Science Frontier Research, 18, 37-58.

[11] Ho, V.B. (2020) Classification of Relativity. Journal of Modern Physics, 11, 535-564. https://doi.org/10.4236/jmp.2020.114036

[12] Ho, V.B. (2021) A Derivation of the Ricci Flow. Journal of Applied Mathematics and Physics, 9, 2179-2186. https://doi.org/10.4236/jamp.2021.99137 